\title{
Phasing methods of tiled-aperture coherent beam combining for high peak power lasers
}

\author{
Chun Peng ${ }^{1,2}$, Xiaoyan Liang ${ }^{1,3,4}{ }^{*}$, Renqi Liu $^{1,2}$, Wenqi $\mathrm{Li}^{1,2,4}$, Ruxin $\mathrm{Li}^{1,3,4}$ \\ ${ }^{1}$ State Key Laboratory of High Field Laser Physics, Shanghai Institute of Optics and Fine Mechanics, \\ Chinese Academy of Sciences, Shanghai 201800, China \\ ${ }^{2}$ University of Chinese Academy of Sciences, Beijing 100049, China \\ ${ }^{3}$ IFSA Collaborative Innovation Center, Shanghai Jiao Tong University, Shanghai 200240, China \\ ${ }^{4}$ School of Physical Science and Technology, ShanghaiTech University, Shanghai 200031, China
}

\begin{abstract}
We demonstrate two type phasing methods for tiled-aperture coherent beam combining for high-power lasers based on the near and far field measurement techniques respectively, a comparison is made.
\end{abstract}

\section{Introduction}

Coherent beam combining ( $\mathrm{CBC}$ ) has been considered as a promising way to scale the output capacity of a single laser system, which breaking through the limitations caused by thermal behaviour of gain medium, optical damage threshold of materials and optical coatings, or detrimental nonlinear effects [1]. Recently, CBC of tiled aperture configuration based on active phasing method has been extended to the generation of high peak power laser pulses by the pursuit of extreme intense conditions owing to its ability to achieve $\mathrm{N}^{2}$ ( $\mathrm{N}$ is the number of the combined beams) times peak intensity in the focal plane [2,3]. For this purpose, the primary but the most challenging issue lies in keeping the zeroth-order phases actively stabilized. In this contribution, we present different phasing methods based on the near and far field measurement techniques respectively to find an appropriate phasing solution for tiled-aperture $\mathrm{CBC}$ in the high peak power regime.

\section{Experiments and results}

The phasing methods were demonstrated on a Ti:Sapphire laser delivering femtosecond pulses at a central wavelength of $800 \mathrm{~nm}$ with a FWHM bandwidth of $15 \mathrm{~nm}$ and a repetition rate of $1 \mathrm{kHz}$. The duration of the laser pulses was $50 \mathrm{fs}$, the diameter was $5 \mathrm{~mm}$, and the energy of a single pulse was approximately $0.5 \mathrm{~mJ}$. Fig. 1(a) and Fig. 2(a) show the experimental setups for two-beam $\mathrm{CBC}$ based on the near-field measurement technique and the far-field measurement technique, respectively. In these two structures, the spatial collimation was realized by mirror mounts and a tip-tilt mirror owing to the near- and farfield distributions. The time domain synchronization was controlled by a piezo-electric transducer (PZT) platform in DL1 by adjusting the optical length of the beam. The

\footnotetext{
* Corresponding author: liangxy@siom.ac.cn
} 
synchronization error was diagnosed by the measurement units and delivered to the processing module, wherein the program calculated the error signal and fed it back to the PZT in real time. Camera 2 is a high-speed monochrome camera with a maximum frame rate of $543 \mathrm{fps}$ at the full resolution of $1696 \times 1704$ pixels, which can be further improved to more than $4 \mathrm{~K}$ fps by reducing the frame resolution. The combined beam pattern was recorded by camera 1 .

In Fig. 1(a), a hybrid phasing method based on the near-field interference fringe (NIFF) technique and single crystal balanced optical cross-correlation (BOC) is used. We realized an adjustable beam combining bandwidth of approximately $100 \mathrm{~Hz}$ (limited by the speed of the PZT) and root-mean-square deviation of approximately $\lambda / 51$ (shown in Fig. 1(b)) for two beam channels with a combining efficiency of $93 \%$.

(a)
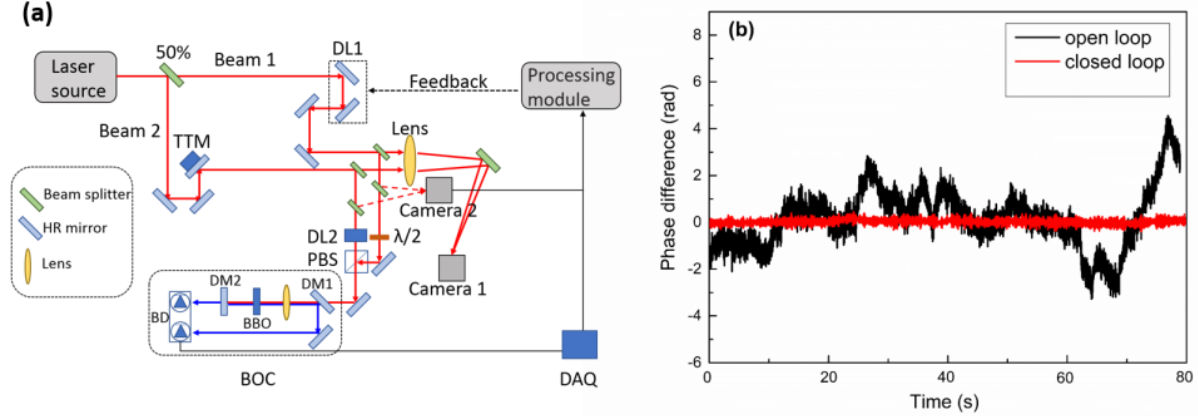

Fig. 1. (a)Experiment setup. BD, balanced photodetector; BOC, balanced optical cross-correlator; DL1/DL2, delay line; DM1, dichroic mirror, AR at $800 \mathrm{~nm}$, HR at $400 \mathrm{~nm}$; DM2, dichroic mirror, $\mathrm{AR}$ at $400 \mathrm{~nm}, \mathrm{HR}$ at $800 \mathrm{~nm}$; PBS, polarization beam splitter; TTM, tip-tilt mirror; BBO, beta barium borate crystal; DAQ, data acquisition card. (b) Phase errors recorded in an open loop and closed loop.

In Fig. 2(a), the phasing technique is based on the far-field interference fringe measurement. The phase error is diagnosed directly from the far-field of the tiled-aperture $\mathrm{CBC}$ where the coherent combination happens. The bandwidth is approximately $100 \mathrm{~Hz}$ which is limited by the speed of PZT. The phase error is controlled with an RMS deviation of $0.157 \mathrm{rad}$ (approximately $1 / 40 \lambda$ ), shown in Fig. 2(b), the coherently combined beam pattern corresponding to a coherent efficiency of $92 \%$.

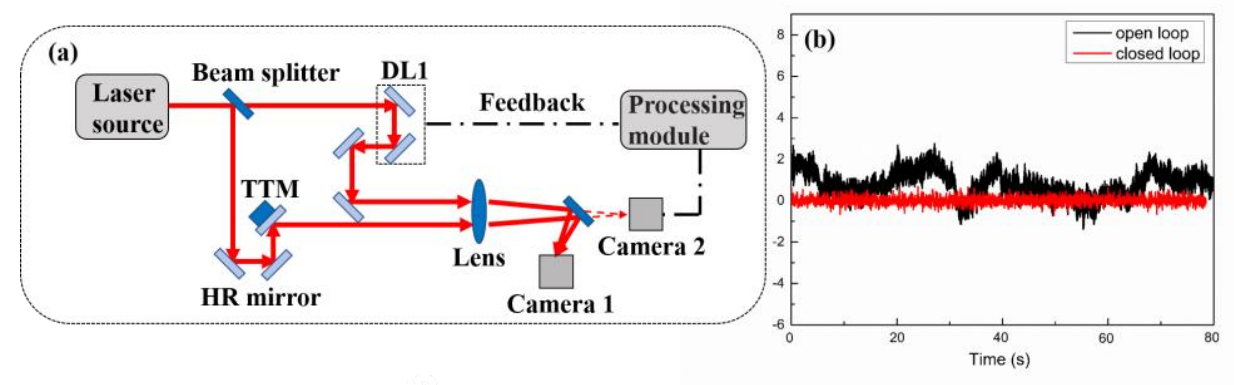

Fig. 2. (a) Experiment setup. DL1 is delay line, HR mirror is high reflected mirror, and TTM is tip-tilt mirror. (b) Phase errors recorded in the open loop and closed loop.

Comparing these two structures, we can see that the phase error of tiled-aperture CBC can be diagnosed from whether the near-field or the far-field. Phasing method based on the near-field measurement is more convenient to adjust and possesses better accuracy. The 
latter one based on the far-field measurement has simpler optical structure but with higher algorithm requirement, the measurement accuracy is also limited by the resolution of Camera 2. But detecting the phase error from the far-field offers a direct observation of the coherent combination effect and realize a full-path error measurement for the reason that the ultra-intense field is created in the focal plane. Moreover, the phase error of tiledaperture can be detected by translated into electrical signal (the BOC [4] and HanschCouillasud detectors [2]) or image signal (interference fringe detector [5]). Combining different type approaches can raise the anti-disturbing capacity of the system.

\section{Conclusion}

To summarized, we present different implementations of phasing method for tiled-aperture $\mathrm{CBC}$ based on the near-field and far-field measurement techniques. In the proof-ofprinciple experiment, all these meet the phase control requirement of femtosecond pulses $\mathrm{CBC}$. The merit and demerit is compared.

\section{References}

1. T. Y. Fan, IEEE J. Sel. Top. Quantum Electron. 11, 567, Jun. (2005).

2. S. Bagayev, V. Leshchenko, V. Trunov, E. Pestryakov, and S. Frolov, Opt. Lett. 39, 6, 1517 (2014).

3. J.-P. Chambaret, O. Chekhlov, G. Chériaux, J. Collier, R. Dabu, P.Dombi, A. M. Dunne, K. Ertel, P. Georges, J. Hebling, J. Hein,C.Hernandez-Gomez, C. Hooker, S. Karsch, G. Korn, F. Krausz, C.Le Blanc, Z. Major, F. Mathieu, T. Metzger, G. Mourou, P. Nickles,K. Osvay, B. Rus, W. Sandner, G. Szabó, D. Ursescu, and K.Varjú, Proc. SPIE 7721, 77211D (2010).

4. S.-W. Huang, G. Cirmi, J. Moses, K.-H. Hong, S. Bhardwaj, J. R. Birge, L.-J. Chen, E. Li, B. J. Eggleton, G. Cerullo, and F. X. Kärtner, Nat. Photonics 5, 475 (2011).

5. C. Peng, X. Liang, R. Liu, W. Li, and R. Li, Opt. Lett. 42, 3960 (2017). 